\title{
Instantaneous positions of microwave solar bursts: Properties and validity of the multiple beam observations
}

\author{
C.G. Giménez de Castro $^{1}$, J.-P. Raulin ${ }^{1}$, V.S. Makhmutov ${ }^{2}$, P. Kaufmann ${ }^{1}$, and J.E.R. Costa ${ }^{1}$ \\ 1 CRAAE: (Mackenzie, INPE, USP, UNICAMP), Instituto Presbiteriano Mackenzie, Rua de Consolação 896, 01302-000, \\ S. Paulo, SP, Brazil \\ 2 P.N. Lebedev Institute RAS, Leninsky Prospect 53, 117924 Moscow, Russia
}

Received May 28; accepted June 9, 1999

\begin{abstract}
The multiple beam technique determine burst sources positions when their angular extent are small compared with the beam shapes. We show for the first time that we can check the above condition with the simultaneous observation using at least four beams. The developed technique is not critically dependent on source shapes. By means of simulations we found that for narrow 1 arcmin long sources the uncertainties in position determination are less than 5 arcsec, and much better for symmetrical sources. The influence of side lobes on source positions determinations was found to be negligible. A qualitative method was developed when data from only three beams are available. Both methods are applied to the analysis of a solar burst observed with multiple beams at $48 \mathrm{GHz}$ with the Itapetinga $13.7 \mathrm{~m}$ antenna. The multiple beam technique also offers the unique advantage to determine flux density irrespectively from the position displacements of the source with respect to the beams, or vice versa.
\end{abstract}

Key words: sun: flares — sun: radio radiation techniques: interferometric

\section{Introduction}

Solar radio observations using large apertures at short microwaves, producing beams smaller than the solar disk, have shown levels of burst activity in millisecond time scales and sensitivity levels of $10^{-2}$ solar flux units (1 s.f.u. $=10^{-22} \mathrm{Wm}^{-2} \mathrm{~Hz}^{-1}$ ) which are three to two orders of magnitude smaller compared to the respective lower limits of usual patrol telescopes. (for example Kaufmann et al. 1976, 1980; Hurford et al. 1979; Butz et al. 1976; Gaizauskas \& Tapping 1980).

Send offprint requests to: C.G. Giménez de Castro
It was shown that for active regions and for bursts occurring on them, which sizes were small compared to the antenna beamwidth, the high sensitivity attained allowed very precise beam pointing, to the level of several arcseconds (Kaufmann et al. 1982).

On the other hand, proposed models of energetic production at the origin of solar flares indicate the crucial importance of the diagnostic of rapid phenomena both in time and space, simultaneously. However, for fast solar burst time developments, producing structures shorter than one second, it is not possible to scan with a single beam the burst source in subsecond time scales, in order to obtain simultaneous informations on intensity and angular positions within the lifetime of a single rapid spike. The existing technique available to obtain high spatial resolution (few arcseconds) is based on aperture synthesis using large interferometric arrays (see for example Marsh \& Hurford 1982; Kundu \& Lang 1985; Kundu et al. 1990; Bastian et al. 1994). Burst time structures can be shorter than $50 \mathrm{~ms}$ which is of the same order of the best time resolution reported so far for VLA solar observations, being most of time much larger than this (e.g. Bastian et al. 1994).

The multiple beam technique, originally introduced by Efanov \& Moiseev (1967), was addressed to this observational problem, allowing at the same time the determination of position and flux with a high time resolution. It uses a focal array system of feed-horns and radiometers placed at the focus of a large antenna. The partially overlapping beams track continuously a solar active center. As a burst occurs, with angular dimension small compared to beamwidth, the instantaneous relationship between the antenna temperatures for the different beams allows the determination of the angular position with very high accuracy, limited by tracking and radio-seeing conditions. The system development was accomplished within an agreement between the solar group operating the Itapetinga $13.7-\mathrm{m}$ radome enclosed radio 


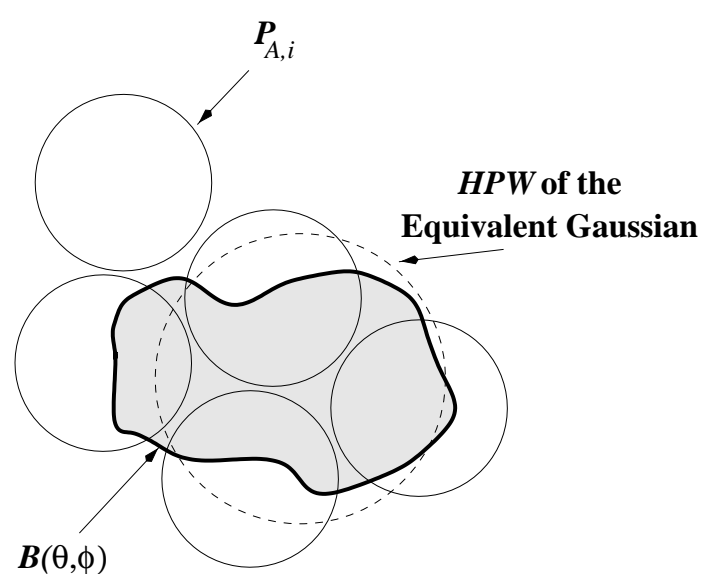

Fig. 1. Five beams (circles) arrangement observing a source with a brightness distribution $B(\theta, \varphi)$ (thick line) which is assumed to have a Gaussian equivalent shape (dashed circle)

telescope, presently at CRAAE in Brazil, and the solar group from the Institute of Applied Physics, IAP, University of Bern in Switzerland. Five $48 \mathrm{GHz}$ feedhorns/radiometers and data acquisition system were built and developed at IAP and the array was placed at the Cassegrain focus of the Itapetinga dish. The beams are partially overlapping to each other by about 1 arcmin, at the individual beams' half power levels (see Fig. 1).

A brief description of the multibeam $48 \mathrm{GHz}$ feedhorns' and radiometers' setup was given by Georges et al. (1989). The first observations at Itapetinga began in 1989. Some aspects of multiple beam observations and the first results obtained were published elsewhere (Herrmann et al. 1992; Costa et al. 1995). In summary, the system achieve a $1 \mathrm{~ms}$ time resolution in the determination of position and flux, with a sensitivity as small as 0.04 s.f.u.

As pointed out above, the multiple beam technique was primarily conceived to study angular positions of solar bursts time structures assumed small compared to the antenna beamwidths. For burst sources components large compared to the beam sizes the multiple beam technique cannot provide positions unequivocally, unless a number of additional assumptions are made. Point-like sources were assumed in the first published analysis (Herrmann et al. 1992, 1997; Costa et al. 1995 and Correia et al. 1995) and definitions of "flux densities" assigned to equivalent point sources producing the measured antenna temperatures for each beam were adopted deriving angular positions for assumed "centroids of burst emissions".

The multiple beams actually observe a burst source which brightness distribution is not known in advance. The antenna temperature for each beam can be expressed as:

$T_{\mathrm{A}, i} \propto \iint_{\Omega} B(\theta, \phi) P_{\mathrm{A}, i} \mathrm{~d} \Omega$

where $P_{\mathrm{A}, i}$ is the beam pattern of the $i$-th receiver and $B(\theta, \phi)$ is the source brightness distribution in space (see Fig. 1). The solution of the system of Eqs. (1) provide an

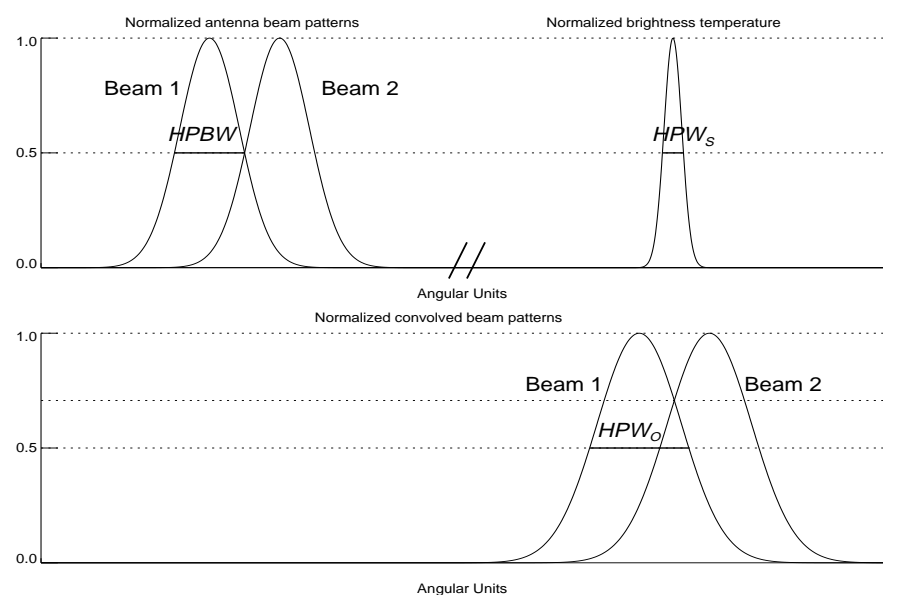

Fig. 2. Schematic representation of the convolution of two identical overlapping Gaussian beams with half power beam width, $H P B W$ (top left) and a Gaussian brightness temperature distribution with a half power width $H P W_{\mathrm{S}}$ (top right). The observed convolved patterns have a half power width $H P W_{\mathrm{O}} \geq$ $H P B W$ (bottom)

angular position, which was called as the position of the centroid of emission. Since $B(\theta, \phi)$ is not known, when applying this method to extended sources the position obtained is just an approximate indication of the direction where the brightness is higher. The time variation of the determined position provides "dynamic maps" of the relative changes in that direction along a certain interval of time. It must be clarified that they are not instantaneous "images" of the bursting site.

The analysis is thus dependent on the assumptions made for the distribution $B(\theta, \phi)$. For example, it is not known in advance if $B(\theta, \phi)$ has a pronounced asymmetry in space, or is built up by multiple sources different in extent and position.

The goal of the present study is to develop methods to check whether the bursts sources are small or large compared to the multiple beams of the array as a necessary precondition for instantaneous position and flux determination. The only assumption made is that $B(\theta, \phi)$ is an axially symmetric function. For sake of simplicity we have adopted a Gaussian. Indeed, once the equivalent source extension is found to be smaller compared to the beams, the source actual shape becomes irrelevant for our purpose to determine position. We discuss pronounced asymmetry in Sect. 2.2. For sources small compared to $H P B W$, sources' positions can be determined unambiguously, and the approximation adopted for $B(\theta, \phi)$ will not produce errors in position bigger than other uncertainties intrinsic to the system. For sources large compared to $H P B W$, positions cannot be determined, unequivocally.

The departing point of the new method takes the realistic observed patterns which are actually the convolution between the antenna beam patterns and the source brightness temperature distribution in space. The observed 
patterns and the brightness distribution are not known in advance (see Fig. 2). Antenna temperatures measured by the different beams can be converted into flux density only for sources small compared to the $H P B W$. It will be shown that using data from at least four independent beams, assuming that the beam shapes and the sources' brightness distributions can be approximated to Gaussian functions, angular extent can be estimated for the burst sources and positions can be calculated for sources small compared to the beam size.

Data from three beams are not sufficient to define burst extent and their positions cannot be calculated unequivocally. Nevertheless we will discuss particular situations for which data obtained with three beams indicate qualitatively whether or not the burst sources are small compared to the beams. When the tests are consistent to small sources, their positions can be calculated using data from only three beams.

Finally we stress that, for sources found to be small compared to the multiple beams' $H P B W$, the technique has the unique advantage to determine their fluxes irrespectively from their positions with respect to the beams, or vice-versa.

\section{Angular positions determination from four beams observations}

\subsection{Basic formalism}

The observed antenna temperature is the convolution between the brightness-temperature distribution of the source $\left(T_{\mathrm{S}}\right)$ and the beam pattern $\left(P_{\mathrm{A}}\right)$. Rewriting Eq. (1) in one dimension (for the sake of simplicity)

$T_{\mathrm{A}}\left(\varphi_{\circ}\right)=\frac{\eta}{\Phi_{\mathrm{A}}} \times \int T_{\mathrm{S}}(\varphi) P_{\mathrm{A}}\left(\varphi-\varphi_{\circ}\right) \mathrm{d} \varphi ;$

where, $\Phi_{\mathrm{A}} \doteq \int P_{\mathrm{A}}\left(\varphi_{\mathrm{A}}\right) \mathrm{d} \varphi$ and $\eta$ is a factor taking into account various system losses, such as the beam and aperture efficiencies and the radome transmission. The main lobe of the beam pattern can be sufficiently well approximated by means of a Gaussian function,

$P_{\mathrm{A}}(\varphi)=\exp \left(-\frac{\varphi^{2}}{2 \sigma_{\mathrm{A}}^{2}}\right) ;$

where the antenna half power beam width $(H P B W)$ is related to $\sigma$ as $H P B W^{2}=-8 \sigma_{\mathrm{A}}^{2} \ln (1 / 2)=2 \sigma_{\mathrm{A}}^{2} \ln (16)$. Assuming for the source brightness temperature distribution also a Gaussian function,

$T_{\mathrm{S}}(\varphi)=T_{\text {S。 }} \exp \left(-\frac{\varphi^{2}}{2 \sigma_{\mathrm{S}}^{2}}\right) ;$

with a half power width, $H P W_{\mathrm{S}}^{2}=-8 \sigma_{\mathrm{S}}^{2} \ln (1 / 2)=$ $2 \sigma_{\mathrm{S}}^{2} \ln (16)$, the convolution of this two functions is straightforward and yields

$T_{\mathrm{A}}\left(\varphi_{\circ}\right)=T_{\mathrm{S} \circ} \sqrt{2 \pi} \mu \exp \left(-\frac{\varphi_{\circ}^{2}}{2\left(\sigma_{\mathrm{A}}^{2}+\sigma_{\mathrm{S}}^{2}\right)}\right) ;$ where $\mu=\sqrt{\sigma_{\mathrm{A}}^{2} \sigma_{\mathrm{S}}^{2} /\left(\sigma_{\mathrm{A}}^{2}+\sigma_{\mathrm{S}}^{2}\right)}$. This result means that a scan over a Gaussian source generates a Gaussian observed brightness temperature distribution with a $\sigma_{\mathrm{O}}$,

$\sigma_{\mathrm{O}}^{2}=\sigma_{\mathrm{A}}^{2}+\sigma_{\mathrm{S}}^{2}$

or, because HPW is proportional to $\sigma$, with an observed half power width

$H P W_{\mathrm{O}}^{2}=H P B W^{2}+H P W_{\mathrm{S}}^{2}$.

Figure 2 illustrates this relationship. The extension of Eq. (5) in the case of the convolution of two axially symmetric Gaussian, is immediate.

Instead of scanning, the multiple beams of the Itapetinga 13.7-m radio telescope are fixed with respect to the source and obtain brightness temperatures pointing to up to five different directions at the same time in order to achieve both a high temporal resolution $(1 \mathrm{~ms})$ and a high precision localization of the direction of emission.

Assuming that the beams transmission coefficients and $H P B W$ are equal for the five receivers, we can write the 2-dimensional Eq. (5) for each beam

$$
\begin{aligned}
T_{\mathrm{A}, i}\left(\varphi_{\circ}, \theta_{\circ}\right)= & 2 \pi \frac{T_{\mathrm{S} \circ} \eta \mu^{2}}{\Phi_{\mathrm{A}}} \\
& \times \exp \left(-\frac{\left(\varphi_{\circ}-\varphi_{\mathrm{A}, i}\right)^{2}+\left(\theta_{\circ}-\theta_{\mathrm{A}, i}\right)^{2}}{2 \sigma_{\mathrm{O}}^{2}}\right) ;
\end{aligned}
$$

where the subscript $i=1,2,3,4,5$ refers to each beam, $\left(\varphi_{\circ}, \theta_{\circ}\right)$ is the displacement in RA and declination between the antenna axis reference direction and the source and $\left(\varphi_{\mathrm{A}, i}, \theta_{\mathrm{A}, i}\right)$ is the displacement between each beam and the antenna axis. Expression (8) is a system of five equations with four unknowns, namely: $T_{\mathrm{So}}, \sigma_{\mathrm{O}}, \varphi_{\circ}$ and $\theta_{0}$. In order to solve it we take four out of the five equations, e.g.: $i=1,2,3$ and 4 and create a reduced system of four equations with four unknowns which is easily solved. We can draw five such reduced set of equations changing the beams used, which can be useful to determine the uncertainties in the solution.

While the interpretation of bursts positions for emission centroids was largely discussed in previous works (e.g. Costa et al. 1995; Correia et al. 1995; Herrmann et al. 1992, 1997) we want here to stress the importance in the determination of $H P W_{\mathrm{O}}$ related to both, the $H P B W$ and the $H P W_{\mathrm{S}}$ by the Eq. (7). The convolution of a very extended source with a beam should result in a big value for $H P W_{\mathrm{O}}$, contrary to the case of a point source, for which we can assume a $H P W_{\mathrm{S}} \approx 0$, resulting in $H P W_{\mathrm{O}} \approx H P B W$.

\subsection{Validity of the method}

We stress that the method finds the instantaneous (1 ms) burst center position, for an isolated source, or the center of an equivalent extended source containing multiple sources emitting simultaneously. In order to assess the accuracy of the position determination, we have carried out 


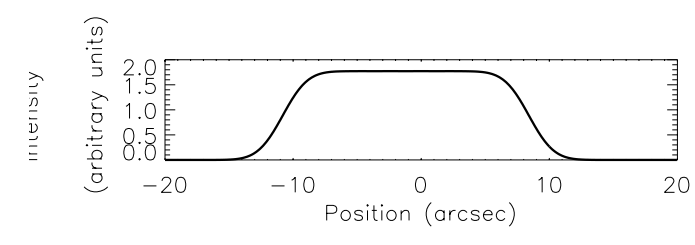

Fig. 3. Example of a longitudinal view of one of the "cigar-like" sources. At half power points it has a length of about 25 arcsec, and a width of 4 arcsec

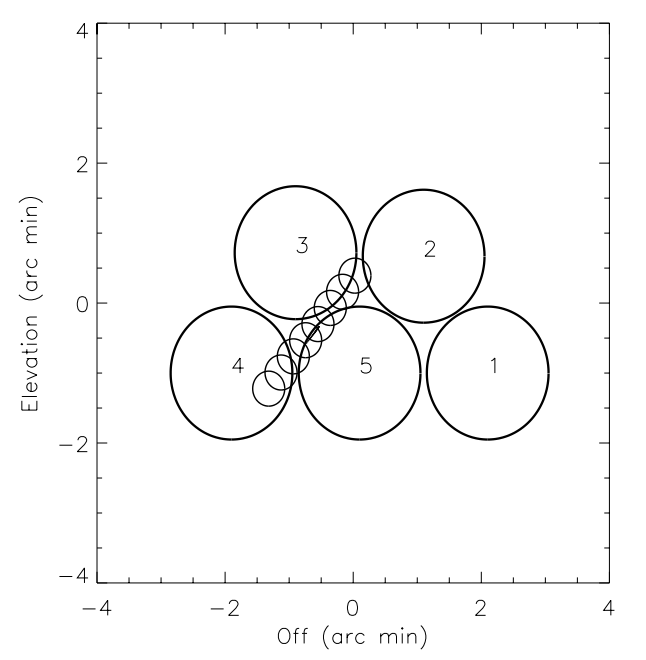

Fig. 4. Antenna beam disposition over a "cigar-like" simulated source. The big circles are the 5 Itapetinga beams at $50 \%$ level. The 8 small circles, between beams 2-3-4-5, build the "cigar" source. This is is the longest source used in our simulations

some simulations. In these simulations the antenna beams are represented by axially symmetric Gaussians. To simulate different sources and sizes, we have used a set of 8 contiguous axially symmetric Gaussians, overlapping each other in a $\mathrm{e}^{-1}$ level. This overlapping creates an extended constant region and produces a "cigar" shape source, with a fixed quotient between principal axes of about 6 (see Fig. 3). Such shapes could be also obtained using other geometrical compositions, with the same final results. We prefer compose Gaussians because of the simplicity in the calculations. It is clear that these "cigars" are no longer the axially symmetric Gaussian sources assumed by the inversion method. In Fig. 4 we show the antenna five beam disposition. In the center of the beams 2-3-4-5, we show one of the "cigars", build up from the 8 small circles. This is the longest source used for the simulations, extending for almost 2.5 arcmin.

In Fig. 5 we show the discrepancy between the real position of the source center of brightness and the computed one in terms of the source length. The discrepancy is computed as the geometrical distance between these two points. The difference, for the longest source $(2.5 \mathrm{arcmin})$ is about 6 arcsec, which is of the same order of the observational uncertainties (Costa et al. 1995); while for a

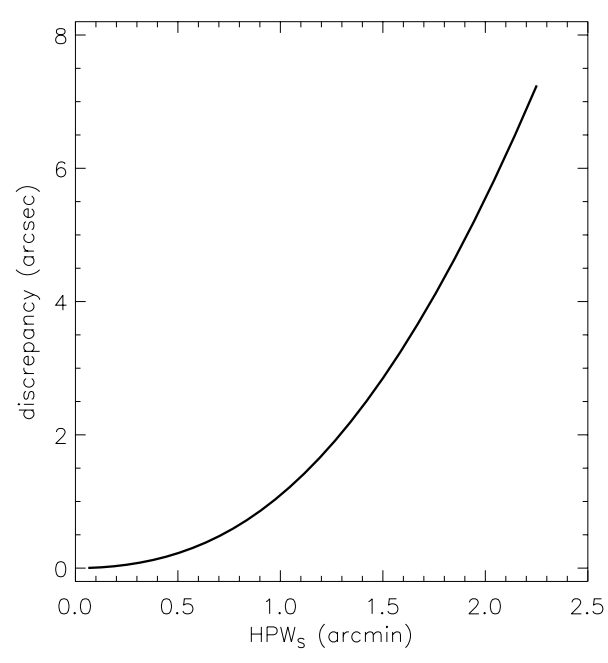

Fig. 5. Discrepancy as a function of source length. Discrepancy is calculated as the geometrical distance between the computed solution and the center of brightness of the "cigar" shaped sources

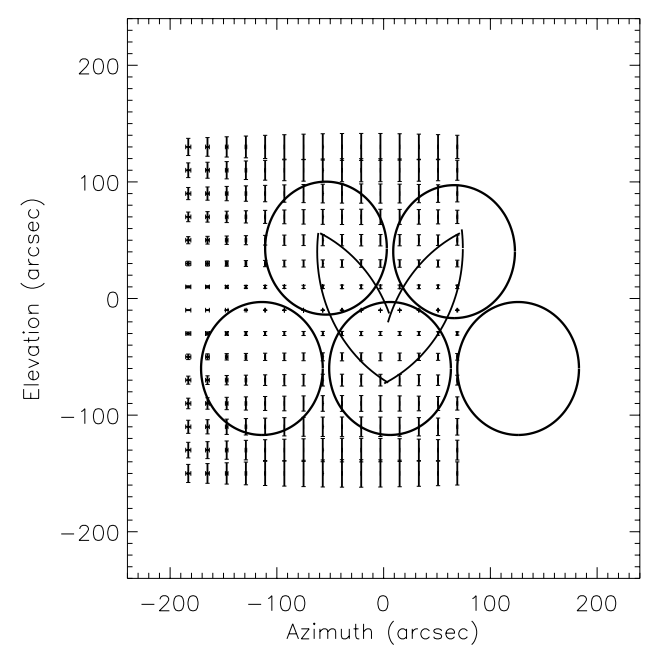

Fig. 6. Position-dependent error in azimuth and elevation over the field of view of channels $2-3-4-5$. Similar results are expected for channels 1-2-3-4. The $6 \%$ level condition is fulfilled inside the area limited by the closed curves

source of 1 arcmin long is reduced to about 1 arcsec. We performed different simulations changing the orientation of the source. In all of them, the results remained in the same range, sometimes, even better.

In another simulation, we took a 1 arcmin $\times 6$ arcsec "cigar-like" source and changed its position over the field of view of channels 2-3-4-5. In Fig. 6 we show the difference in azimuth and elevation between the computed and the real position. Continuous bars indicate position discrepancies. The maximum error in elevation is almost 24 arcsec, while the minimum is near 0 arcsec. In azimuth, the discrepancies are negligible over all locations. 


\subsubsection{Side lobes influence on position determination}

Side lobes may influence the position determination by incrementing the antenna temperature in some channels, where side lobe contribution might be added. This increment could be of the order of $3 \%$ of a main beam level (Herrmann et al. 1992). It is easy to simulate this excess by artificially incrementing the level to some channels. We have carried one simulation where we exceeded that level and applied up to $10 \%$ increment to 1,2 , and 3 out of the 4 channels used to compute positions. The discrepancy in the worst case ( 3 artificially incremented channels), for a 2.5 arcmin long source in the center of the four beams, is 12 arcsec. For a 1 arcmin source length the discrepancy falls to about 3 arcsec.

The assumed Gaussian beam mimetize the central part of the main beam down to the level of $3 \%(-15 \mathrm{db})$ of the peak. Directions outside of this $3 \%$ level on both sides of the main lobe (including its wings) are considered unknown and may contain comma or side lobes with a maximum gain of $3 \%$ of the main beam. A condition to be fulfilled if we want to have non-contradictory solutions, is to have the sources inside the main lobe over the $3 \%$ level. We can check this condition taking quotients between antenna temperatures and assuming the worst situation. This is when one beam observes the source near its $3 \%$ level and, at the same time, the beam which has the highest antenna temperature observes the same source at the farthest point respect to its beam center. In that case the highest antenna temperature beam will be near its $50 \%$ level. The quotient between these two antenna temperatures will be $3 / 50=0.06 \equiv 6 \%$. Thus, our sufficient condition is that 4 out of the 5 beams, have simultaneously antenna temperature quotients, respect to the highest one, bigger than $6 \%$. In Fig. 6 we show the area where this condition is fulfilled, which matches the area where the uncertainties of the method are smaller compared with the observational uncertainties described in Costa et al. (1995).

In summary, we have shown with simulations that, as far as the condition of the above mentioned $6 \%$ ratio is fulfilled, the position determination is very precise (below or similar to the observational uncertainties) and very robust when considering side lobe effects. In practice we have shown that the influence of side lobes on position determination is negligible even for extreme cases of long asymmetric sources. Better knowledge of beam shapes and side lobes will not improve considerably the accuracy of the method in comparison to the observational uncertainties.

Finally, we conclude that, for a source of up to 1 arcmin long, irrespectively of the shape and provided that the above described $6 \%$ criterion is fulfilled, the uncertainty in position determination is smaller than or of the same order of the observational uncertainties (i.e. around or below 5 arcsec).

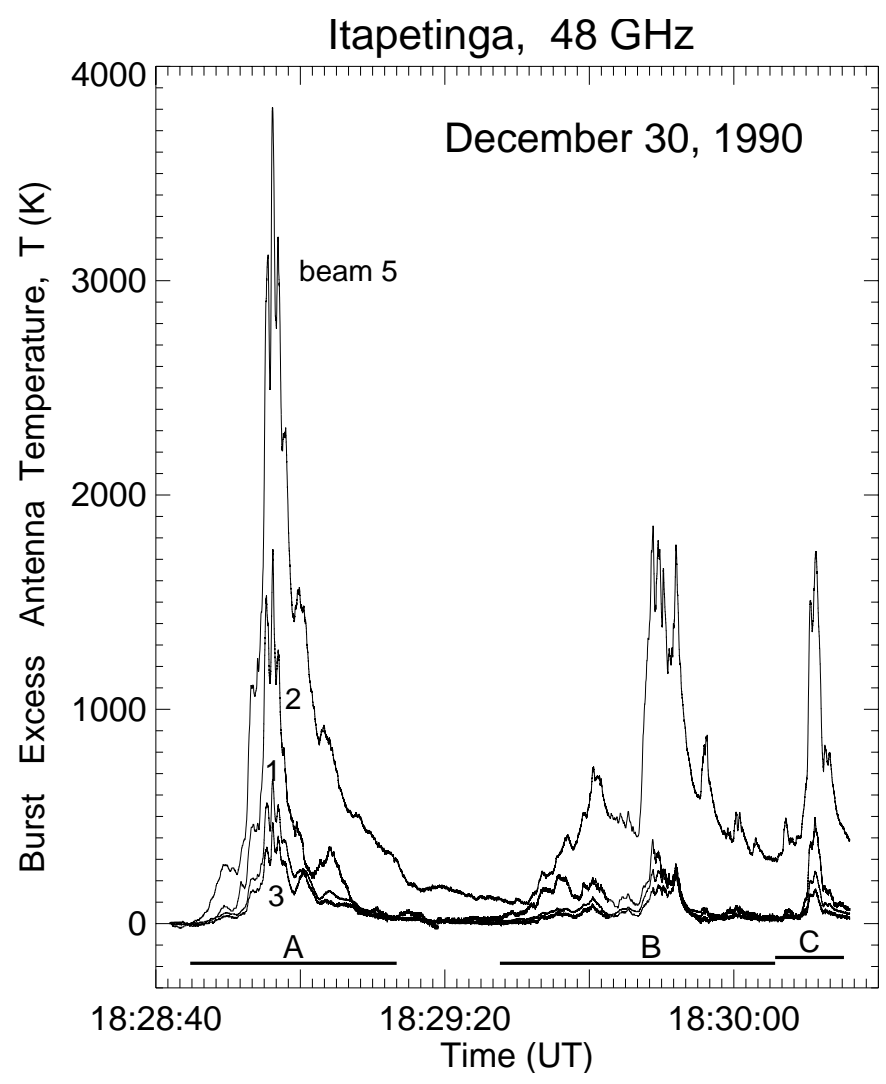

Fig. 7. Time profiles of the $48 \mathrm{GHz}$ antenna temperatures during the solar burst of December 30, 1990, as recorded by four beams of the Itapetinga antenna (beam 1, 2, 3 and 5; integration time $8 \mathrm{~ms}$ ). The horizontal bars labeled by A, B, C indicate the time intervals for which fine time structures are shown in Fig. 4 (a, b, c)

\section{Application to the flare of December 30, 1990}

To demonstrate the method developed here we concentrate on the solar burst of December 30, 1990. It is one of the very few events obtained at Itapetinga with more than three beams and it was analyzed in part by Costa et al. (1995). This event was associated with a SN/M1.2 solar flare which started at 18:29 UT at the heliographic position S09 W47 in the active region AR 6624 (SGD 1990). Figure 7 displays the $48 \mathrm{GHz}$ radio emission time profiles, obtained by four beams $(1,2,3,5)$ of the Itapetinga antenna, between 18:28:40 and 18:30:20 UT. We have selected three time periods (A, B and C; see horizontal lines at the bottom) during which major time structures are observed, and for which the inferred convoluted beam angular extent and sources positions will be computed using the analytical solution of the system of Eqs. (8).

Figure 8 shows detailed antenna temperature time profiles for the periods $\mathrm{A}, \mathrm{B}$ and $\mathrm{C}$ observed by the beam 5 (top). The values of the observed convolved beam, $H P W_{\mathrm{O}}$ (bottom) are calculated with the data of four beams as described in Sect. 2. Data plotted was taken with $8 \mathrm{~ms}$ time resolution except for the beginning of the event (up to 

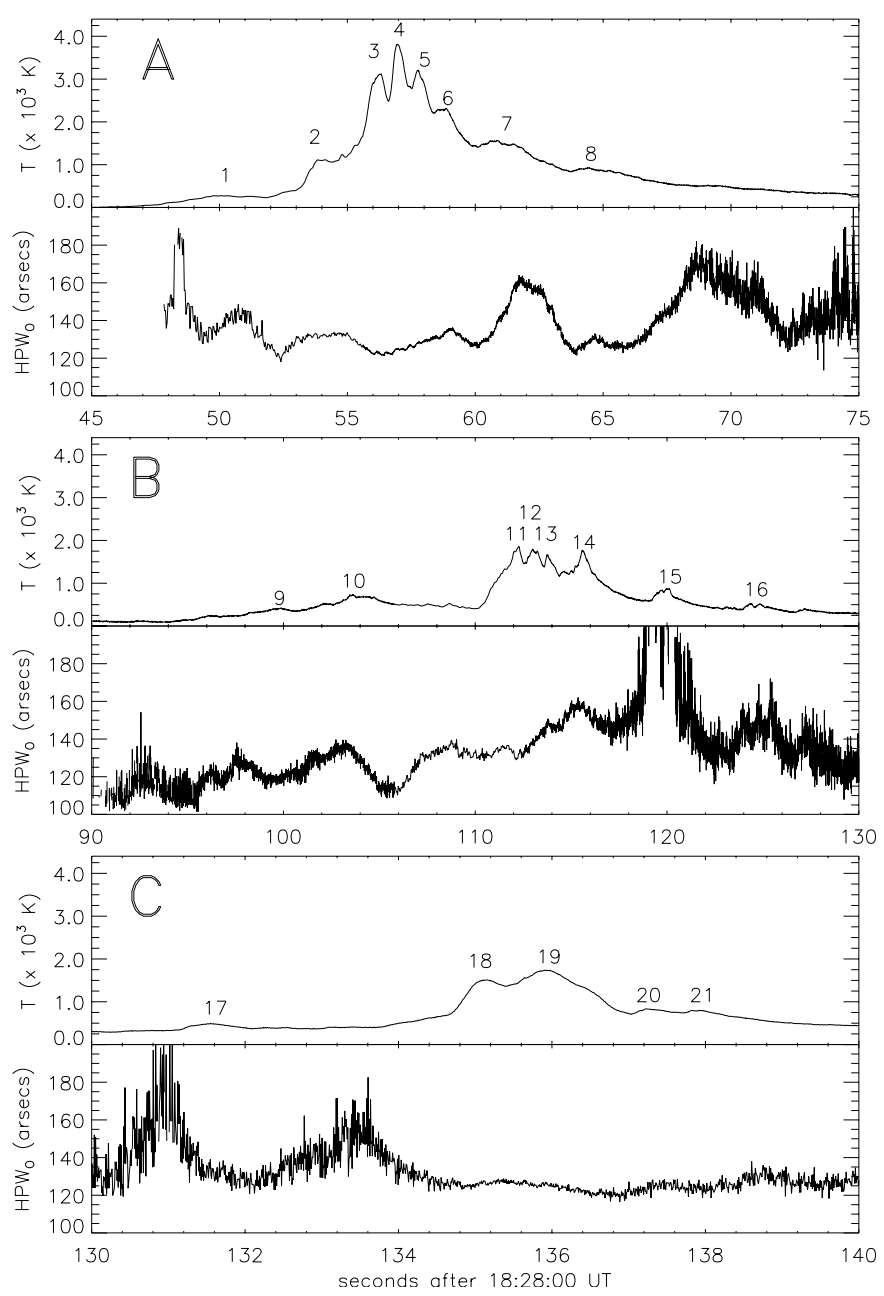

Fig. 8. $48 \mathrm{GHz}$ radio emission time profiles (top) as recorded by beam 5 and temporal evolution of the estimated values of the $H P W_{\mathrm{O}}$ (bottom) for time intervals $\mathrm{A}, \mathrm{B}$ and $\mathrm{C}$, shown in Fig. 7. The values of the $H P W_{\mathrm{O}}$ have been calculated assuming symmetrical Gaussian brightness distribution

58 sec after 18:28:00 UT) where the time resolution was $32 \mathrm{~ms}$. The "noise" patterns in the plots of $H P W_{\mathrm{O}}$ are the result of the system temperature fluctuations, which become more pronounced for small burst signal levels, at least in one of the four beams. We note that the variations in $H P W_{\mathrm{O}}$ are considerably more pronounced than the noise, suggesting a genuine variation with time of the source (or source complex) angular extension. Due to the beamshape uncertainty a point source convolved with our beams might produce a $H P W_{\mathrm{O}}$ in the range from 102 to 136 arcsec. All the structures, except 15, seen in the event have $H P W_{\mathrm{O}}$ between 130 and 140 arcsec, which indicates that sources are small compared with our beams and then their positions can be determined unequivocally.

Figure 9 shows the calculated positions for peaks labeled in Fig. 8 (top). The size of the crosses corresponds to two times the standard deviation of positions determined within $400 \mathrm{~ms}$ intervals around the peaks. The positions

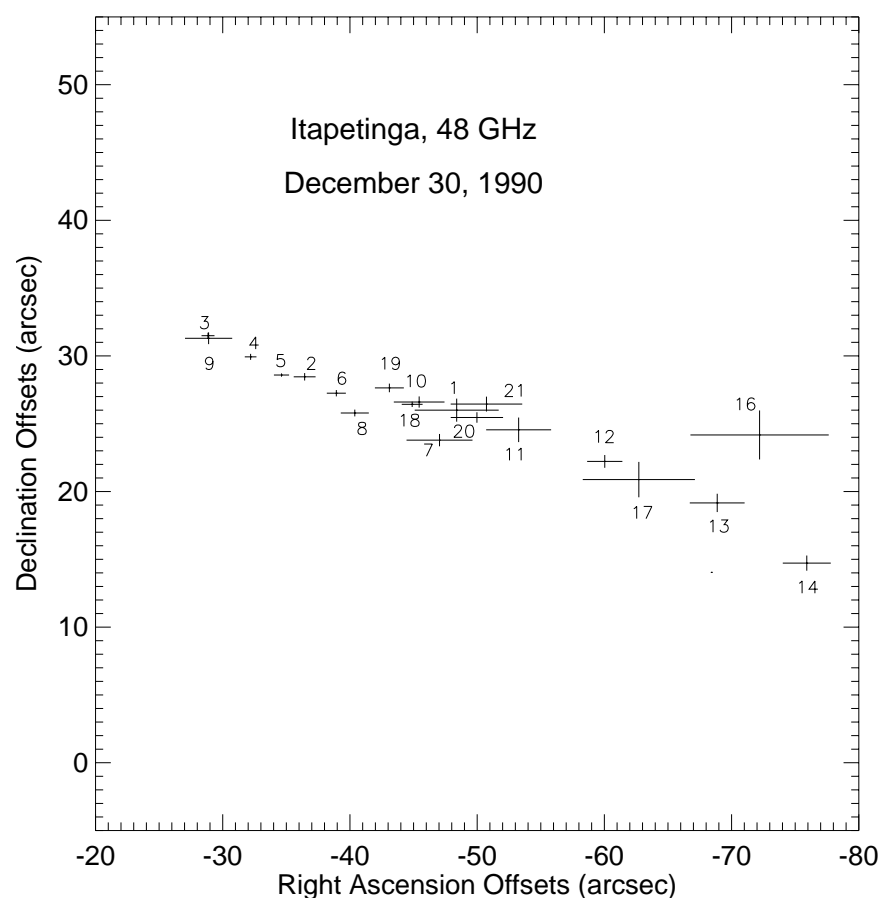

Fig. 9. Mean positions of the sources for the peaks labeled from 1 to 21 in Figs. 8 (a, b, c). The mean value is obtained by averaging the data during $400 \mathrm{~ms}$ around each peak. The size of the crosses correspond to two times the standard deviation. Peak 15 position was not determined because some antenna temperatures are bellow the $6 \%$ level

of the peaks lay in a NE-SW strip, about 10 arcsec wide, 40 arcsec long, with peaks from structure A (Figs. 7, 8a) located eastwards. Position of peak 15 cannot be estimated because some of the antenna temperatures are below the limit of the $6 \%$ level respect to the maximum observed temperature.

\section{Qualitative method with three beams observations}

Data obtained from only three beams are not sufficient to determine unambiguously the radio burst position, unless the assumption of a small (compared to beam) emitting source is made.

A qualitative method was developed based on the idea that the source angular extent should be related to antenna temperature ratios obtained with different beams. These ratios lead to a contrast criterion, which should increase for smaller sources. That is to say: if an emitting source is small compared to the $H P B W$, the contrast should be higher than for an extended emitting region at the same location. For three beams observations, we can define a contrast parameter $K$ as:

$K=\ln \left(\frac{T_{\mathrm{H}}}{T_{\mathrm{L}}}\right)+\ln \left(\frac{T_{\mathrm{I}}}{T_{\mathrm{L}}}\right)=\ln \left(\frac{T_{\mathrm{H}} T_{\mathrm{I}}}{T_{\mathrm{L}}^{2}}\right) ;$

where $T_{\mathrm{H}}, T_{\mathrm{I}}$, and $T_{\mathrm{L}}$ are the high, intermediate and low observed antenna temperatures respectively. 

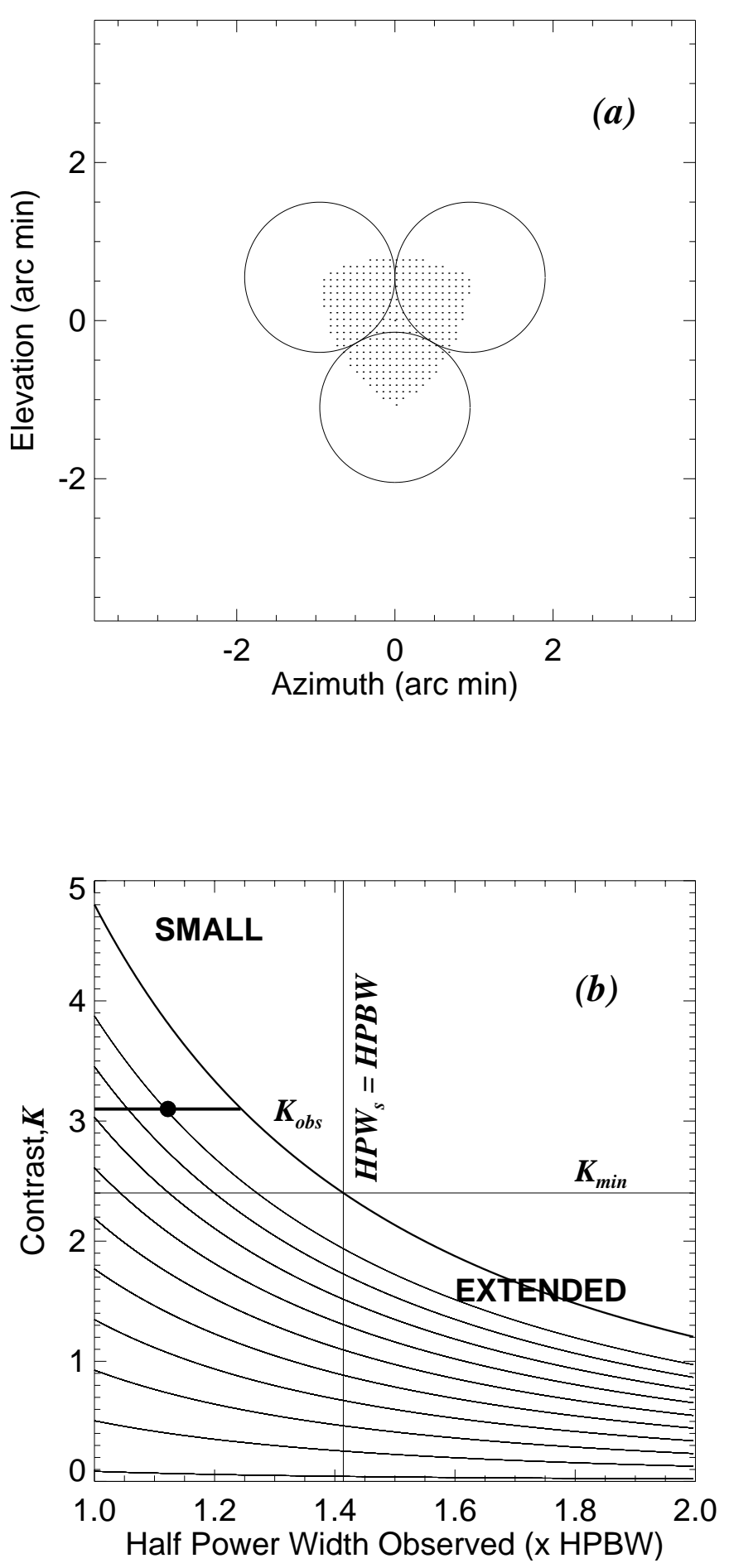

Fig. 10. a) Three beams disposition at $48 \mathrm{GHz}$. The dotted area shows the location of radio sources for which there is no signal exceeding $-15 \mathrm{db}$ from sidelobes. b) Variations of the contrast $K$ as a function of $H P W_{\mathrm{O}}$, for different location of the emitting sources within the dotted area. The line $K=K_{\text {min }}$ defines 2 regions of contrast values. Only $K$ belonging to the upper part indicates a small source, compared to the $H P B W$. The thick horizontal line represents the range of uncertainty in $H P W_{\mathrm{O}}$ for a given $K_{\text {obs }}$. The dot in this line represents the mean value
We have computed the expected contrast $K$ for different positions within three beams (Fig. 10a) and different source sizes, assuming Gaussian source shapes. The source locations (dotted area in Fig. 10a) have been chosen such that, at each location the antenna temperatures $T_{\mathrm{H}}, T_{\mathrm{I}}$, and $T_{\mathrm{L}}$ satisfy the condition:

$0.06 T_{\mathrm{H}}<T_{\mathrm{L}}<T_{\mathrm{I}}<T_{\mathrm{H}}$

assuring that the data have no contamination by signals from sidelobes. The contrast $K$ has been computed at each location, and for different values of $H P W_{\mathrm{O}}$ in the range $H P B W-2 \times H P B W$, corresponding to a point emitting source and an emitting source of extension $H P W_{\mathrm{S}} \sim \sqrt{3} \times H P B W$, respectively. Here we have assumed that the beam size is 1.9 arcmin (see Sect. 2). The results are shown in Fig. 10b. Each curve shows, for a given emitting source location, the evolution of $K$ versus $H P W_{\mathrm{O}}$ (in units of $H P B W$ ). The limiting curve (thick line in the plot) corresponds to the highest $K$ for each source size and was computed analytically for the actual geometry. The condition $H P W_{\mathrm{S}}=H P B W\left(H P W_{\mathrm{O}}=\sqrt{2} \times H P B W\right)$, represented by the vertical line, defines a minimum value $K_{\min }$ above which the size of the emitting source is necessarily smaller than the $H P B W$. Figure 10b shows clearly that if $K<K_{\min }$, there is an ambiguity in comparing the source size with the $H P B W$. The bottom left quadrant shows small (compared to $H P B W$ ) emitting sources located near the origin 0 , where the contrast is below the limit $K_{\min }$ which does not allow to distinguish between small (compared to $H P B W$ ) and extended emitting sources. In this case, source positions using three beams observations will not be computed.

In summary, the qualitative method should be used as follows with three beams observations: i) compute a contrast value $K_{\text {obs }}$, ii) refer to Fig. 10 b to compare $H P W_{\mathrm{O}}$ and $H P B W$ (same as comparing $K_{\text {obs }}$ to $K_{\min }$ ), iii) if $H P W_{\mathrm{O}} \leq \sqrt{2} \times H P B W$ the position of the burst is computed, if not the position of the burst is not estimated. Our uncertainty in the half power width observed is the range defined by $H P W_{\mathrm{O}}=H P B W$ and the $H P W_{\mathrm{O}}$ which corresponds to $K=K_{\text {obs }}$ in the limiting curve. In Fig. 10b we show, for a given $K_{\mathrm{obs}}$, the range of uncertainty. In order to compute positions we adopt $H P W_{\mathrm{O}}$ equals to the mean value of this range.

As an illustration we have applied the qualitative method to the 30 December 1990 event, which has been observed with four beams (see previous section), allowing an unambiguous determination of the burst position as well as its instantaneous angular extent. For the qualitative test we used the antenna temperatures observed with beams 2,3 , and 5 . Figure 11 shows the absolute discrepancies as large dots, computed as angular distances, between positions computed with the method described in Sect. 2 and the qualitative method for the time periods A, B, C (Fig. 7). Angular positions for peaks 1, 12, 13, 14 and 16 are not computed because have contrast lower 
Discrepancies in angular positions computed with 4 and 3 beams
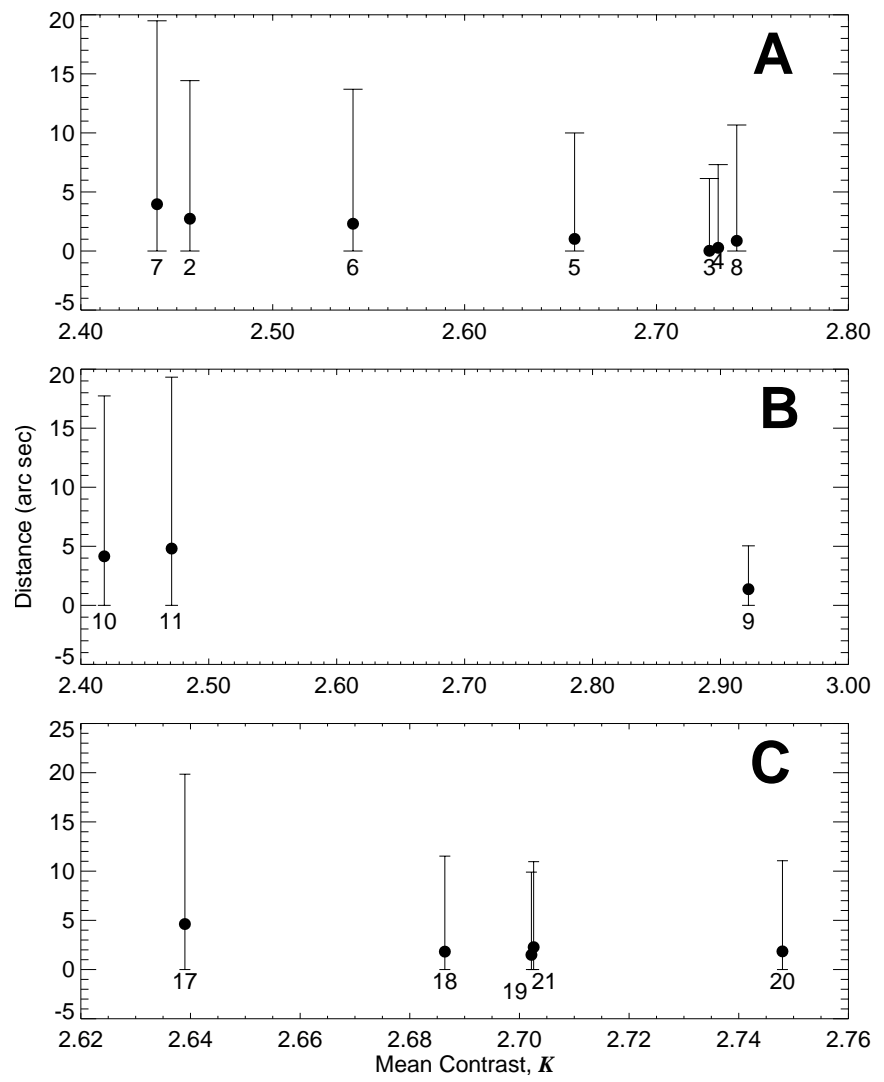

Fig. 11. Discrepancies measured as angular distances between the positions computed with 4 beams and with beams 2-3-5 (qualitative method) for the time structures showed in Fig. 7) of the burst on 30 December 1990 in terms of mean contrast $K$. Dots are the absolute discrepancies. Vertical bars show the uncertainties

than $K_{\text {min }}$; while angular position for peak 15 is not considered because the condition given by the Eq. (10) is not fulfilled. In all the computed cases, discrepancies are less than 5 arcsec and the general trend is: for larger contrast $K$ the discrepancy becomes smaller. In Fig. 11 vertical bars show the uncertainties due to the range in $H P W_{\mathrm{O}}$ allowed by each $K_{\text {obs }}$. Here also the general trend is an inverse relation between contrast and uncertainty.

\section{Discussion}

In Sect. 2 of this paper we have shown that the solution of the system of Eqs. (8) leads to instantaneous estimates of the burst radio source extent and its position. The determination that the burst extent is small compared to the $H P B W$ is a necessary condition to determine their position. This technique has been applied to the flare of December 30 1990, 18:28:00 UT, observed at $48 \mathrm{GHz}$ with the Itapetinga multibeam system.

The time structure A of the December 301990 event (Figs. 7 and 8 peaks 1 to 8 ) has already been studied as-
Discrepancies in angular positions computed by Costa et al (1995) and this work

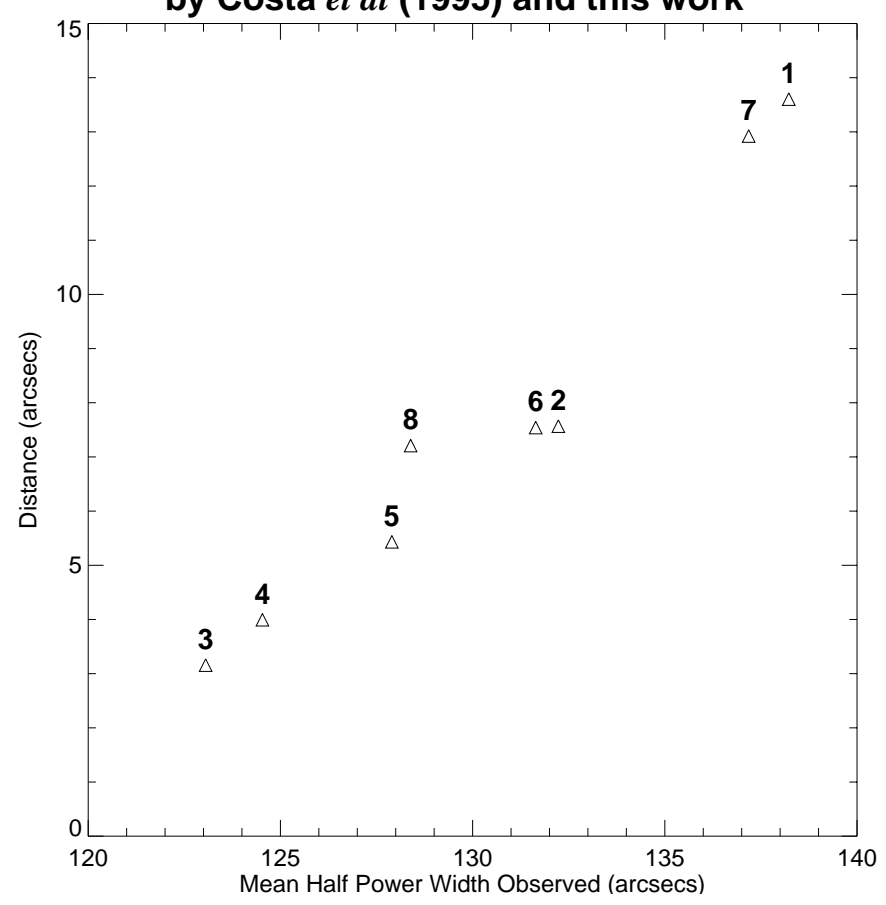

Fig. 12. Discrepancies measured as angular distances between positions computed by Costa et al. (1995) and the new method using four beams for the time structures labeled in Fig. 8 of the burst on 30 December 1990 in terms of mean $H P W_{\mathrm{O}}$. Larger discrepancies are found for larger source angular extents $H P W_{\mathrm{O}}$

suming that the radio emission was produced by a point source (Costa et al. 1995). We compared the position calculations by the two methods which are displayed in Fig. 12. We have plotted the positions for peaks $1-8$ computed using the previous method and positions determined using the present method. The disagreement in position for the two methods are less than about 5 arcsec, for the most intense peaks, 3, 4 and 5. It becomes more pronounced for the less intense peaks, 1, 7 and 8 , being about 15 arcsec. These discrepancies result from the fact that the previous method assume $H P W_{\mathrm{O}}=H P B W$ and constant throughout all the event duration; while the present method computes $H P W_{\mathrm{O}}$ at each time.

Finally the time variations of the burst angular extent, as shown in Fig. 4 for Gaussian equivalence, might deserve further studies as for its validity as inputs for physical interpretation which is beyond the scope of the present work.

\section{Conclusions}

The necessary condition to unequivocally determine position and flux with a multibeam technique is that the source is smaller than the beam sizes. We have developed a method to assess the burst source angular extent 
which requires data from four beams simultaneously. The qualitative method for three beams provides similar results, except for particular burst positions with respect to the beams. The method is based on the actually observed beams which are the convolution of the source brightness distribution to the beams patterns. The only assumptions made, for simplicity, are that the antenna beam patterns and the sources brightness distribution can be approximated to Gaussian functions. By means of simulations, we have shown that for sources of shape different than axially symmetric Gaussians, the expected error in position made by the inversion method, is of the same order or smaller than the observational uncertainties (about 5 arcsec), for a source of up 1 arcmin long. We also found that a sufficient "a priori" criterion to compute position is to have a greater than $6 \%$ quotient between the lowest and the highest antenna temperatures. Finally, we demonstrate to be negligible any possible side lobe effect for levels as large as $10 \%$.

The method was compared to the previously used approach, for the $48 \mathrm{GHz}$ observation of the solar burst of 30 December 1990, 18:30 UT. This event has three successive major time structures with superimposed finer features. The half power width observed has shown variations with time, and indicated sources' angular extent small or comparable to the $H P B W$. The positions were then calculated. The difference in position computed by the previous method (Costa et al. 1995) ranges from 5 to 15 arcsec, increasing as the $H P W_{\mathrm{O}}$ increases.

The qualitative method to be used when burst data are available only for three beams can provide the angular extent and position determinations. The method is based on the ratio of antenna temperatures for the three beams, the contrast criterion, setting possible conditions for the burst size and position computations. This can be used successfully when the observed contrast is sufficiently high to define unambiguously that the source is small compared with the $H P B W$. This method was compared to the results obtained using four beams for the 30 December 1990 event.

The use of multiple beam technique for the instantaneous analysis of bursts angular extents and positions is a powerful tool for the investigation of the flaring processes, the locations and time development of different emissions. Assumptions about the physical nature of the radio emissions received might be added to the analysis of the data. For example, in certain cases it may be possible to separate burst emission components due to fast (non-thermal) and to slow (thermal) mechanisms, and multiple beam data analysis done accordingly. However this discussion is beyond the scope of the present paper, and will be the subject of other studies.

The new methods will have important applications in the analysis of the $48 \mathrm{GHz}$ bursts recorded at Itapetinga in the period 1989-1994, most of them obtained only with three beams. A new $48 \mathrm{GHz}$ system is presently being developed, and future observations will greatly benefit with the use of these improved methods. Another important application is directed to the new Solar Submm-wave Telescope, the SST project (Kaufmann et al. 1996) which, at its lower frequency of $210 \mathrm{GHz}$, will have an array of three beams separated at their $50 \%$ levels, and a fourth beam separated at the $10 \%$ level from other two beams.

Source equivalent angular extent are derived here just as a tool to check whether or not the sources are small compared to the antenna beams for the purpose of the position determination. However this magnitude might have potential for physical interpretation. This is being studied separately.

Acknowledgements. We wish to thank an anonymous referee whose comments were very helpful for the improvement and the clarity of the present study. We also gratefully acknowledge fruitful comments and suggestions given by A. Magun and K. Arzner from the Institute of Applied Physics (University of Bern, Switzerland).

Partial support for the present work was given by the Fundação de Amparo à Pesquisa do Estado de São Paulo (FAPESP, Brasil) through grants Nos. 93/3321-7, 96/06956-1 and 94/5957-9, and by Conselho Nacional de Desenvolvimento Científico e Tecnológico (CNPq, Brasil) through grant No. 150087/96-9.

CRAAE, Centro de Rádio-Astronomia e Aplicações Espaciais, is a joint center between Mackenzie, INPE, USP and UNICAMP.

\section{References}

Bastian T.S., Nitta N., Kiplinger A.L., Dulk G.A., 1994, in: Proc. Kofu Symposium, Enome S. and Hirayama T. (eds.), NRO report No. 360, 199

Butz M., Hirth W., Furth E., Harth W., 1976, in: Sond. Kleinhenbacker Berichte 19, 345

Correia E., Costa J.E.R., Kaufmann P., Magun A., Herrmann R., 1995, Solar Phys. 159, 143

Costa J.E.R., Correia E., Kaufmann P., Magun A., Herrmann R., 1995, Solar Phys. 159, 157

Efanov V.A., Moiseev I.G., 1967, Izv. KRAO 38, 149

Gaizauskas V., Tapping K.F., 1980, ApJ 241, 804

Georges C.B., Schaal R.E., Kaufmann P., Magun A., 1989, in: Proc. 2nd International Microwave Symposium, Rio de Janeiro, p. 447

Herrmann R., Magun A., Costa J.E.R., Correia E., Kaufmann P., 1992, Solar Phys. 142, 157

Herrmann R., Magun A., Kaufmann P., Correia E., Costa J.E.R., Machado M.E., Fishman G.J., 1997, A\&A 317, 232

Hurford G.J., Marsh K.A., Zirin H., Kaufmann P., Strauss F.M., 1979, BAAS 11, 678

Kaufmann P., Iacomo Jr., Koppe E.H., Santos P.M., Schaal R.E., 1975, Solar Phys. 45, 189

Kaufmann P., Strauss F.M., Raffaelli J.C., Opher R., 1980, in: Proc. of Solar Terrestrial Publications, Donnelly R.F. (ed.), NOAA, ERL, Vol. 3, 78, 389 
Kaufmann P., Strauss F.M., Schaal R.E., Laporte C., 1982, Kundu M.R., White S.M., Gopalswamy N., Biejing J.H., Solar Phys. 78, 389 Hurford G.J., 1990, ApJ 358, L69

Kaufmann P., Costa J.E.R., Correia E., Magun A., Arzner K., Marsh K.A., Hurford G.J., 1982, ARA\&A 20, 497

Kämpfer N., Rovira N., Levato H., 1996, Proc. of CESRA Solar Geophysical Data Bulletin: 1990, ERL, NOAA, Boulder Kundu M.R., Lang K.R., 1985, Sci 228, 9 Colorado, U.S.A. 